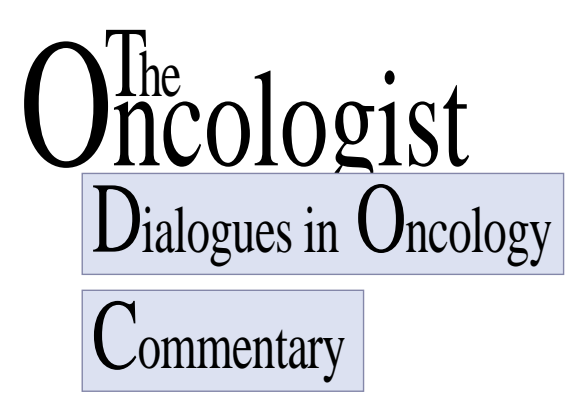

\title{
Emerging New Opportunities for Patients with Hepatic Metastases from Colorectal Cancer or Primary Hepatocellular Cancer
}

\author{
H.M. Pinedo, C.J. van Groeningen \\ Department of Medical Oncology, University Hospital Vrije Universiteit, Amsterdam, The Netherlands
}

Treatment of primary tumors of the liver as well as of liver metastases arising from other primary tumors has been a challenge for many disciplines in oncology [1, 2]. Both diagnoses used to be considered a lethal verdict, but things are definitely changing for those patients without extrahepatic disease.

While liver metastases from colorectal carcinoma are the leading cause of cancer morbidity and mortality in Western society, hepatocellular carcinoma (HCC) ranks number one in morbidity/mortality in many Asian and African countries.

It is only a few decades ago that, for both conditions, therapeutic nihilism was the rule. Great efforts have been undertaken to develop better medical (i.e., chemotherapy) therapies. For HCC this has completely failed, while chemotherapy for metastatic colorectal cancer is also often of limited success, despite the recent development of novel active antineoplastic agents.

The diagnosis of HCC is often made at a time before metastases have developed, while in colorectal carcinoma metastases are confined to the liver in a significant proportion of the patients. Therefore, there is a relative advantage in comparison with other neoplasms that often harbor disease at multiple sites.

In the past 5-10 years, resection of one to three liver metastases resulted in a long-term patient survival of $20 \%$ [3]. More recently, Kemeny et al., showed that these patients may benefit from adding adjuvant postoperative hepatic arterial chemotherapy [4]. However, many patients with large unilateral disease were excluded from surgery as they frequently appeared to have additional, often deeply located non-resectable metastases in the contralateral lobe. Similarly, resection of primary HCC is often not feasible, due to the large tumor volume, the presence of multiple primary tumors, or concomitant liver cirrhosis.

In this issue of The Oncologist, Curley [5] as well as Bilchik et al., [6] elegantly present their experience with radiofrequency ablation (RFA) in both primary $\mathrm{HCC}$ and in colorectal liver metastases. Although both authors discuss the feasibility of the percutaneous approach, between the lines it appears that each of them favor the laparoscopic or open surgical approach which affords a better view of the liver and the peritoneal surface, limits liver blood flow during RFA whenever required, and this provides the opportunity to implant an arterial infusion device, if appropriate.

We totally agree with both authors that resection is the prime goal of the treatment in these patients. Nonetheless, RFA is a great asset to be added to a multidisciplinary approach. This technique, which is still in a developmental phase, is associated with fewer complications when compared with other methods such as cryosurgical ablation (CSA), injection of ethanol, heat ablation using microwave coagulation therapy, or laser-induced thermotherapy. Of

Correspondence: Herbert M. Pinedo, M.D., Ph.D., Head of Oncology, Free University Hospital, Department of Medical Oncology, De Boelelaan 1117, Amsterdam, 1081 HV, The Netherlands. Telephone: 31-20-444-4342; Fax: 31-20-444-4081; e-mail: pinedo@worldaccess.nl CAlphaMed Press 1083-7159/2001/\$5.00/0 
note, the results presented by the authors have been achieved without adjuvant hepatic arterial chemotherapy.

The size limits of the tumors permitting RFA may differ, with Curley mentioning a maximum of 5-6 $\mathrm{cm}$ and Bilchik referring to a maximum diameter of $3-4 \mathrm{~cm}$. However, both authors agree that the limits are moving upwards as newer RFA devices become available. While resection is often out of the question for lesions lying close to the hepatic artery, portal vein and branches of the hepatic vein, RFA (not CSA) can still be applied in certain instances. It appears that the endothelium is resistant to RFA but not to CSA-induced damage. Margin-free resections cannot be obtained for lesions located at such sites, but lesions have been destroyed successfully by RFA. Both authors mention the advantage of temporary occlusion of arterial blood flow while applying RFA as the temperature within the lesion will reach much higher levels without a tendency to drop immediately after reaching the peak level. Amazingly, temperatures up to $90^{\circ} \mathrm{C}$ can be achieved at the target site. Also, newer devices are rapidly becoming available, which may provide therapeutic opportunities for patients with lesions up to $10 \mathrm{~cm}$.

Although long-term observations are still not available, this technique will definitely give the surgeon a helping hand, and offers the patient better prospects. Patients for whom surgical resection of a large lesion was contraindicated because of additional lesions in the contralateral lobe, have become candidates for surgery because of RFA. Bilchik recommends routine placement of an arterial infusion pump in all patients who undergo resection and/or ablation, while Curley still views this as experimental. In our institute we have recently applied a technique for hepatic arterial infusion chemotherapy consisting of the percutaneous placement of a catheter through the subclavian artery, with the tip located in the hepatic artery and the proximal end connected with a subcutaneously placed access device (Port-A-Cath) in the upper chest wall. As the percentage of recurrences will be reduced to approximately $50 \%$ with the multimodality approach, a catheter which can be removed in the outpatient clinic at the end of the adjuvant chemotherapy will offer great advantages for this group of patients.

In summary, the two key articles in this issue of The Oncologist focus on the value of RFA, a technique that offers great benefit to both patients with metastatic colorectal cancer and those with primary HCC. It is advisable to keep this technique in the experienced surgical hands associated with referral centers where they have experience with the treatment of this group of challenging patients. The standard approach may ultimately become surgical resection, RFA (with temporary occlusion of blood flow), followed by hepatic arterial infusion chemotherapy with fluoropyrimidines and, in the near future, in combination with other cytotoxic agents.

\section{REFERENCES}

1 Kemeny NE, Ron IG. Liver metastases. Curr Treat Options Gastroenterol 1999;2:49-57.

2 Ulmer SC. Hepatocellular carcinoma. A concise guide to its status and management. Postgrad Med 2000;107:117124.

3 Yoon SS, Tanabe KK. Surgical treatment and other regional treatments for colorectal cancer liver metastases. The Oncologist 1999;4:197-208.
4 Kemeny N, Huang Y, Cohen AM et al. Hepatic arterial infusion of chemotherapy after resection of hepatic metastases from colorectal cancer. N Engl J Med 1999;341:2039-2048.

5 Curley S. Radiofrequency ablation of malignant liver tumors. The Oncologist 2001;6:14-23.

6 Bilchik AJ, Wood TF, Allegra DP. Radiofrequency ablation of unresectable hepatic malignancies: lessons learned. The Oncologist 2001;6:24-33.

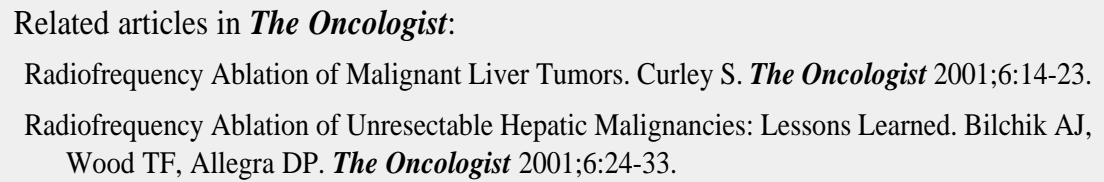

